\title{
Specific Gravity Calculation of H2So4 Using Automation
}

\author{
Avadhut Bhagwat, Siddhesh Sahasrabudhhe, Sachin Dhore,Prof. S.B.Dhonde \\ Researchers, University of Pune AISSMS's Institute of Information Technology, Electronics Dept. Pune-411001, \\ Maharashtra, India. \\ University of Pune AISSMS's Institute of Information Technology, Electronics Dept.Pune-41 1001, Maharashtra \\ India
}

\begin{abstract}
The general idea behind the paper is to mix two liquids i.e. h2so4 (sulphuric acid) \& distilled mineral water in such a desired proportion so that the output liquid will contain some acidity, which accordingly will be required and used for certain industrial \& consumer purpose. The role of automation using programmable logic controller is that to maintain the specific gravity of the output product as per required. Since this chemical mixing process is exothermic reaction, we use temperature sensor to measure and maintain the temperature inside the mixing plant.

Here in this automation project, the main hardware Programmable Logical Controller used is of Siemens s7-300 and the Human Machine Interface used is of delta make, dop-bs7115 and the software's used are simatic manager and dopsoft respectively. The output liquid solution obtained has its application in batteries used in cars, inverters, generators and also used for electroplating purpose.

Keywords: PLC, $\mathrm{HMI}_{1} \mathrm{H}_{2} \mathrm{SO}_{4}$, Distilled Mineral Water.
\end{abstract}

\section{Introduction}

The main objective of this paper is to develop an automated system for maintaining specific gravity by calculating the amount of sulphuric (H2SO4) acid and distilled mineral water to be mixed in the plant. Initially, this mixing used to be entirely worker based process. Due to this the specific gravity amount accuracy was not precisely maintained. There were number of human errors which produced non-prescribed time delay and improper working functionality. Hence there was a need of specific system that could replace human work having high accuracy and continuous working capability.

The devices like programmable logical controller (PLC), human machine interface (HMI) have proved to be highly accurate, re-programmable and possessing easy user interface, leading towards building highly successful automated plant. The added advantage of these systems is the wide flexibility for interfacing.

In the applications like specific gravity maintenance where multiple acidic solutions are involved for producing output liquid solution, any tolerance is not acceptable. So this criterion is completely satisfied. Specific gravity is the ratio of the density of a substance to the density (mass of the same unit volume) of a reference substance. Apparent specific gravity is the ratio of the weight of a volume of the substance to the weight of an equal volume of the reference substance. Generally the reference substance is water for liquid since the specific gravity of water is 1 . Since our project is of specific gravity maintenance, it is important to consider parameters such as specific gravity, as it is a ratio of densities, is a dimensionless_quantity and it varies with temperature and pressure. Specific gravity is commonly used in industry as a simple means of obtaining information about the concentration of solutions of various materials such as brines, hydrocarbons, sugar solutions (syrups, juices, honeys, brewers etc.) and acids. In our automation project specific gravity is maintained by calculating the amount of heat generated. Specific gravity and temperature are inversely proportional to each other. Since the chemical process of mixing is an exothermic process, the temperature evolved is monitored and thus ultimately specific gravity is maintained.

An exothermic reaction is a chemical_reaction that releases energy in the form of light or heat. Here in our project, we are monitoring heat evolved. For a given temperature a particular amount of specific gravity remains constant. Hence using temperature sensor such as pt100, the amount of heat evolved i.e. temperature of the chemical process is calibrated and thus the specific gravity is maintained accordingly. If the temperature fluctuates then accordingly the amount of liquids to be mixed are either increased or decreased to maintain a subtle temperature thus ultimately calculating and maintaining the specific gravity of the output liquid.

\section{Literature survey}

A number of experimental methods for determining the specific gravities of solids, liquids, and gases have been devised. A solid is weighed first in air, then while immersed in water; the difference in the two weights, according to Archimedes' principle, is the weight of the water displaced by the volume of the solid. If the solid is less dense than water, some means must be adopted to fully submerge it, e.g., a system of pulleys or 
a sinker of known mass and volume. The specific gravity of the solid is the ratio of its weight in air to the difference between its weight in air and its weight immersed in water.

Two methods are commonly used for determining the specific gravities of liquids. One method uses the hydrometer, an instrument that gives a specific gravity reading directly. A second method, called the bottle method, uses a "specific-gravity bottle," i.e., a flask made to hold a known volume of liquid at a specified temperature (usually $20^{\circ} \mathrm{c}$ ). The bottle is weighed, filled with the liquid whose specific gravity is to be found, and weighed again. The difference in weights is divided by the weight of an equal volume of water to give the specific gravity of the liquid. For gases a method essentially the same as the bottle method for liquids is used. Specific gravities of gases are usually converted mathematically to their value at standard temperature and pressure.

Earlier Project Workings For Specific Gravity Calculation: Specific gravity as the ratio of the mass of a volume of a material at a stated temperature to the mass of the same volume of distilled water at a stated temperature. Specific gravity has a variety of important applications in hot mix asphalt engineering. Perhaps most importantly specific gravity is used in determination of the percent air voids. The general equation for determining specific gravity assuming the material and water are at the desired temperature is:

Specific gravity $=\{[$ mass of material $/$ volume of material $] /$ density of water $)$

\section{A. Objective of the system}

\section{Objectiveandspecification}

The basic objective of our industrial paper is to maintain specific gravity of the solution in the mixer by controlling maintaining temperature inside the mixer. Since, the mixing of the two liquids is an exothermic process; the temperature given out by the chemical reaction is then monitored and controlled thus controlling the specific gravity of the mixed output liquid.

\section{B. Specification of the system}

Automation process mainly consists of interfacing of PLC's and HMI.[2] PLC is a hardware device used for logical control by executing logical programs, programmed by the user. PLC is a flexible programmable device which can handle multiple operations at a single instant of time. HMI is a hardware interface panel used for logical control as well as display of the proposed work. PLC acts as a processor and HMI acts as the monitoring device of an automated system. In our project, we are interfacing PLC and HMI for controlling actuator valves, pumps, motors, drives and flow meters. PLC holds primary side control while HMI indicates final output. The sulphuric acid liquid and distilled mineral water are to be mixed in fixed proportion by controlling pumps and actuator valves, checking the exact value on flow meters, maintaining the temperature at prescribed level as well as cooling and final output liquid production. These processes are made feasible due to control of the above automated devices. PLC mainly controls physical devices and HMI indicates content, requirement, current status of the output liquid. [1]

[B] Physical Devices used are: Flow transmitter for $\mathrm{H}_{2} \mathrm{SO} 4$, Flow transmitter for $\mathrm{H}_{2} \mathrm{SO} 4$, Temp. Sensor for Coolant inlet temperature, Temp. Sensor for Coolant outlet temperature ,Dilute H2SO4 Outlet Temperature, Pressure Transmitter for the Coolant, Coolant Inlet Flow Control Valve, Coolant Outlet On/Off Valve, H2O Tank Outlet Flow Control Valve,H2SO4 Tank Outlet Flow Control Valve .

[C] HMI: Dop-B Series Dop-Bbs7115 Delta Make [5]

[D] PLC: Siemens make S7-300, [1]

[E] Interface Cable between PLC \& HMI: Pearl Automation Made, [1]

Panel Hardware Sheet Metal Form: Eldon-Cs Series,

Switchgears: Schneider Electrical Made.[4]

Software Used: In This project we are using simatic-Manager Software For Programming PLC. The simatic Manager manages all data belonging to a Siemens PLC automation project, regardless of the target system in which they are implemented. We have used "Dop-Soft" software for Programming HMI. 


\section{Design Of The System}

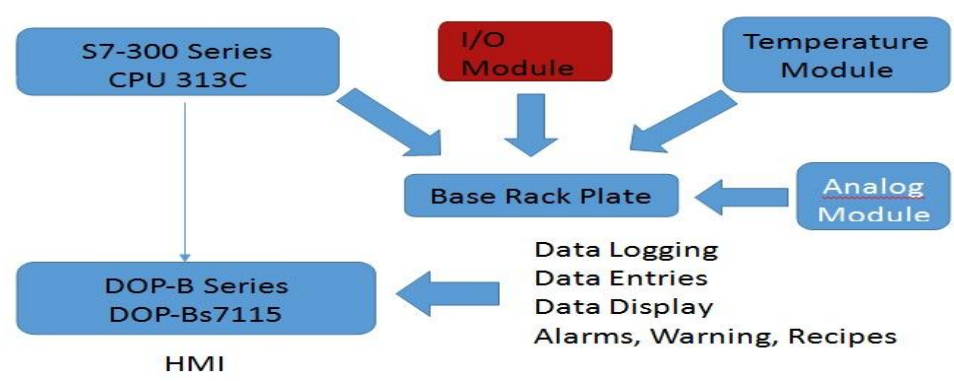

1. (Block Schematic)

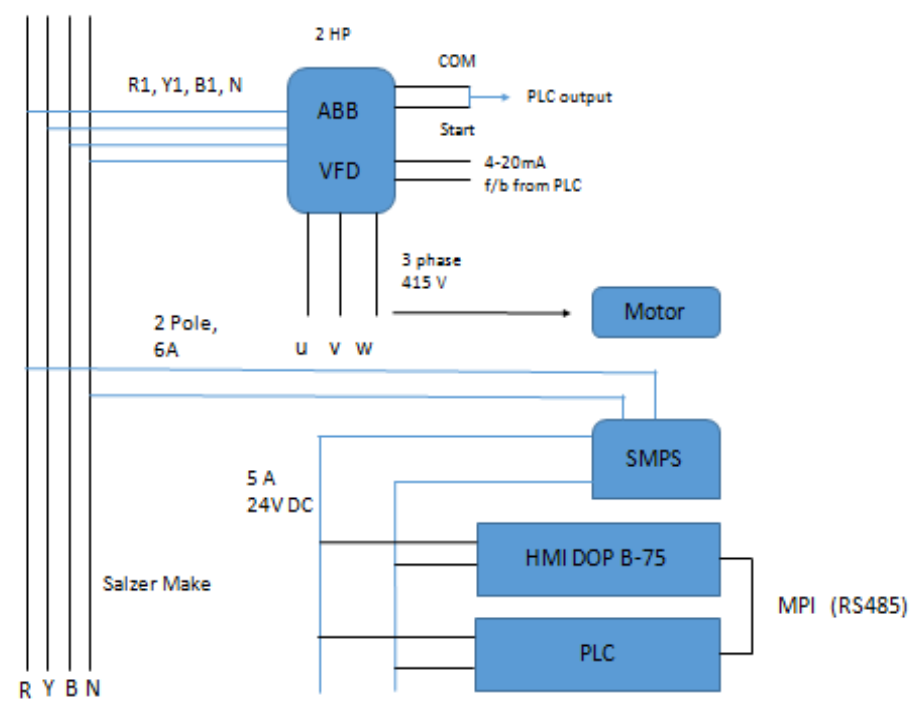

2. (Connection Schematic)

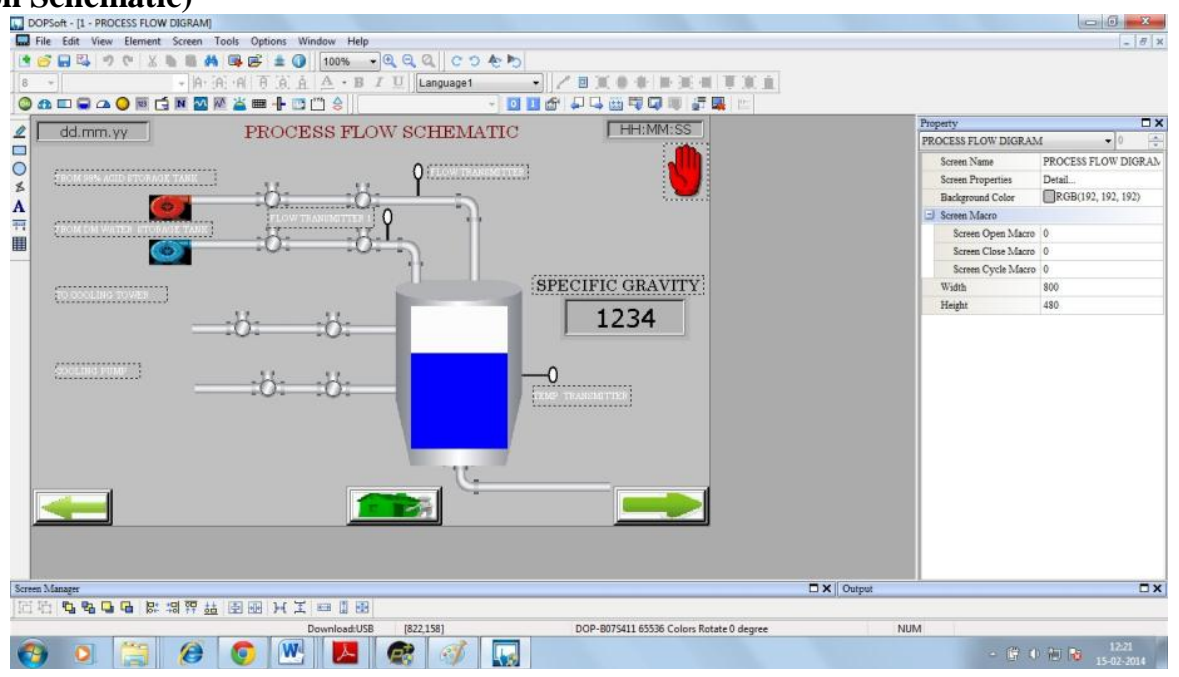

3. (HMI Programming - Process Schematic)

\section{A. Conclusion}

\section{Conclusion And Future Scope}

We have used automation process that can be used to calculate specific gravity of solution by mainly using PLC \& HMI for generating accurate results and smooth control. From this paper, we have covered Basic use of PLC and HMI's in automation process which is used for measurement of specific Gravity of H2SO4 solution. This process of measurement has proved itself to be a very unique one in normal Processes that are used in Specific Gravity calculation. 


\section{B. Future scope}

- Boilers feed water, Textiles, Pharmaceuticals, Chemicals, Breweries, Swimming pools, Potable water, Hospitals, Automobile, and Battery, Fertilizers

- Ion Exchange Plants
○ Softener
$\circ$ Industrial DM Plant
- Two Stage \& Multi Stage DM Plants
- Mix Bed Demineralizer
$\circ$ De-Gasifiers
- Caution Polisher
- Manual/Automatic Plants

[1]. Siemens S-7 300 Manual

\section{References}

[2]. Introduction to PLC by Gary Dunning

[3]. ABB VFD Manual

[4]. Schneider MCB Manual

[5]. HMI DOP-Soft Manual.

[6]. M. H. Rashid, "Power Electronics Circuits Devices and Applications", PHI, 3rd edition, 2004, New Delhi

[7]. Gopal K. Dubey, "Fundamental of Electrical Drives", Narosa publishing House

[8]. Curtis Johnson; "Process Control Instrumentation Technology"; Prentice Hall of India Pvt. Ltd.; 7th Edition 